\title{
ERRATUM
}

\section{Die Phytosanitäre Grenzkontrollstelle Frankfurt/Main-Flughafen stellt sich vor}

\section{Karl Gese}

Online veröffentlicht: 28 Februar 2007

(C) Springer-Verlag 2007

Gesunde Pflanzen (2006) 58 (4): 201-204, S. 202

Im Abschnitt „Die Importe über die PhGKSt Frankfurt/M.“ muss es richtig heißen:

Im Jahr 2005 wurden durch die PhGKSt Frankfurt/M. 27.922 Sendungen pflanzlichen Ursprungs kontrolliert.

Die Online-Version des Originalartikels befindet sich unter: http://dx.doi.org/10.1007/s10343-006-0135-x

K. Gese (浭)

Regierungspräsidium Giessen, Pflanzenschutzdienst Hessen,

Schanzenfelstr. 8, 35578 Wetzlar, Germany

E-Mail: gesek@ulf.hessen.de

Tel.: +49-641-3035225

Fax: +49-641-3035104 